\title{
GAMMA-RAY EXCESS FROM A STACKED SAMPLE OF HIGH- AND INTERMEDIATE-FREQUENCY PEAKED BLAZARS OBSERVED WITH THE MAGIC TELESCOPE
}

\author{
J. Aleksić ${ }^{1}$, L. A. Antonelli ${ }^{2}$, P. Antoranz ${ }^{3}$, M. Backes ${ }^{4}$, C. Baixeras ${ }^{5}$, J. A. Barrio ${ }^{6}$, D. Bastieri ${ }^{7}$, \\ J. Becerra González ${ }^{8}$, W. Bednarek ${ }^{9}$, A. Berdyugin ${ }^{10}$, K. Berger $^{10}$, E. Bernardini ${ }^{11}$, A. Biland ${ }^{12}$, O. Blanch ${ }^{1}$, \\ R. K. BocK $^{7,13}$, G. Bonnoli ${ }^{2}$, P. Bordas ${ }^{14}$, D. Borla Tridon ${ }^{13}$, V. Bosch-Ramon ${ }^{14}$, D. Bose ${ }^{6}$, I. Braun ${ }^{12}$, T. Bretz ${ }^{15}$, \\ D. BritzGer ${ }^{13}$, M. Camara ${ }^{6}$, E. Carmona ${ }^{13}$, A. Carosi ${ }^{2}$, P. Colin ${ }^{13}$, S. Commichau ${ }^{12}$, J. L. Contreras ${ }^{6}$, J. Cortina ${ }^{1}$, \\ M. T. Costado ${ }^{8,16}$, S. Covino ${ }^{2}$, F. Dazzi ${ }^{17,26}$, A. De Angelis ${ }^{17}$, E. De Cea del Pozo ${ }^{18}$, R. De los Reyes ${ }^{6,27}$, B. De Lotto ${ }^{17}$, \\ M. De Maria ${ }^{17}$, F. De Sabata ${ }^{17}$, C Delgado Mendez ${ }^{8,28}$, M. Doert ${ }^{4}$, A. Domínguez ${ }^{19}$, D. Dominis Prester ${ }^{20}$, D. Dorner ${ }^{12}$, \\ M. Doro ${ }^{7}$, D. Elsaesser ${ }^{15}$, M. ERrando ${ }^{1}$, D. Ferenc ${ }^{20}$, M. V. Fonseca ${ }^{6}$, L. Font ${ }^{5}$, R. J. García LóPez ${ }^{8}, 16$, M. GarczarCZyK ${ }^{8}$, \\ M. Gaug ${ }^{8}$, N. Godinovic ${ }^{20}$, D. Hadasch ${ }^{18}$, A. Herrero ${ }^{8,16}$, D. Hildebrand ${ }^{12}$, D. HöHne-Mönch ${ }^{15}$, J. Hose ${ }^{13}$, D. Hrupec ${ }^{20}$,

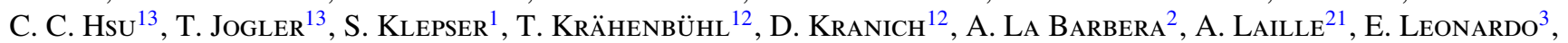

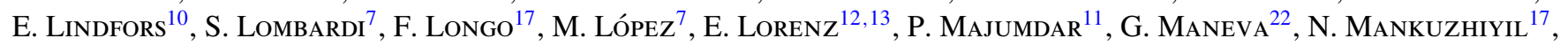 \\ K. Mannheim ${ }^{15}$, L. Maraschi ${ }^{2}$, M. Mariotti ${ }^{7}$, M. Martínez ${ }^{1}$, D. Mazin ${ }^{1}$, M. Meucci ${ }^{3}$, J. M. Miranda ${ }^{3}$, R. Mirzoyan ${ }^{13}$,

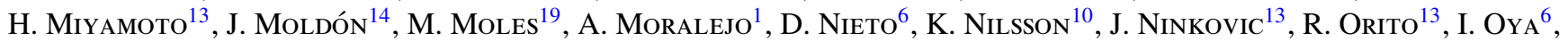 \\ S. Paiano ${ }^{7}$, R. Paoletti ${ }^{3}$, J. M. Paredes ${ }^{14}$, S. Partini ${ }^{3}$, M. Pasanen ${ }^{10}$, D. Pascoli ${ }^{7}$, F. Pauss ${ }^{12}$, R. G. Pegna ${ }^{3}$, \\ M. A. Perez-Torres ${ }^{19}$, M. Persic ${ }^{17,23}$, L. Peruzzo ${ }^{7}$, F. Prada ${ }^{19}$, E. Prandini ${ }^{7}$, N. Puchades ${ }^{1}$, I. Puljak ${ }^{20}$, I. Reichardt ${ }^{1}$,

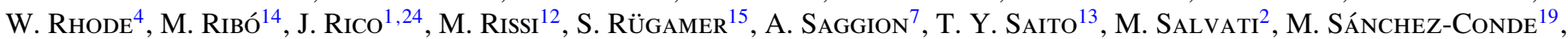 \\ K. Satalecka ${ }^{11}$, V. Scalzotto ${ }^{7}$, V. Scapin ${ }^{17}$, C. Schultz ${ }^{7}$, T. Schweizer ${ }^{13}$, M. Sha Yduk ${ }^{13}$, S. N. Shore ${ }^{25}$, \\ A. Sierpowska-Bartosik ${ }^{9}$, A. Sillanpä̈̈ ${ }^{10}$, J. SitareK $^{9,13}$, D. Sobczynska ${ }^{9}$, F. Spanier ${ }^{15}$, S. Spiro ${ }^{2}$, A. Stamerra ${ }^{3}$, \\ B. Steinke ${ }^{13}$, J. C. Struebig ${ }^{15}$, T. Suric ${ }^{20}$, L. Takalo ${ }^{10}$, F. Tavecchio $^{2}$, P. Temnikov ${ }^{22}$, T. Terzic ${ }^{20}$, D. Tescaro ${ }^{1}$, \\ M. Teshima ${ }^{13}$, O. Tibolla ${ }^{15}$, D. F. Torres ${ }^{18,24}$, H. Vankov ${ }^{22}$, R. M. Wagner ${ }^{13}$, Q. Weitzel ${ }^{12}$, V. Zabalza ${ }^{14}$, \\ F. ZANDANEL ${ }^{19}$, AND R. ZANIN ${ }^{1}$ \\ ${ }^{1}$ IFAE, Edifici Cn., Campus UAB, E-08193 Bellaterra, Spain \\ ${ }^{2}$ INAF National Institute for Astrophysics, I-00136 Rome, Italy \\ ${ }^{3}$ Dipartimento di Fisica, Università di Siena and INFN Pisa, I-53100 Siena, Italy \\ ${ }^{4}$ Fakultät für Physik, Technische Universität Dortmund, D-44221 Dortmund, Germany \\ ${ }_{6}^{5}$ Facultat de Fisica, Universitat Autònoma de Barcelona, E-08193 Bellaterra, Spain \\ ${ }^{6}$ Grupo de Fisica Altas Energias, Universidad Complutense, E-28040 Madrid, Spain \\ ${ }^{7}$ Dipartimento di Fisica, Università di Padova and INFN, I-35131 Padova, Italy \\ ${ }^{8}$ Inst. de Astrofísica de Canarias, E-38200 La Laguna, Tenerife, Spain \\ ${ }^{9}$ Division of Experimental Physics, University of Łódź, PL-90236 Lodz, Poland \\ ${ }^{10}$ Tuorla Observatory, University of Turku, FI-21500 Piikkiö, Finland \\ ${ }^{11}$ Deutsches Elektronen-Synchrotron (DESY), D-15738 Zeuthen, Germany \\ ${ }^{12}$ ETH Zurich, CH-8093 Zurich, Switzerland \\ ${ }^{13}$ Max-Planck-Institut für Physik, D-80805 München, Germany \\ ${ }^{14}$ Facultat de Fisica, Universitat de Barcelona (ICC/IEEC), E-08028 Barcelona, Spain \\ ${ }^{15}$ Fakultät für Physik und Astronomie, Universität Würzburg, D-97074 Würzburg, Germany; hoehne@ astro.uni-wuerzburg.de \\ ${ }^{16}$ Depto. de Astrofisica, Universidad, E-38206 La Laguna, Tenerife, Spain \\ ${ }^{17}$ Dipartimento di Fisica Sperimentale, Università di Udine and INFN Trieste, I-33100 Udine, Italy \\ ${ }^{18}$ Institut de Ciències de l'Espai (IEEC-CSIC), E-08193 Bellaterra, Spain \\ ${ }^{19}$ Inst. de Astrofísica de Andalucía (CSIC), E-18080 Granada, Spain \\ ${ }^{20}$ Croatian MAGIC Consortium, Institute R. Boskovic, University of Rijeka and University of Split, HR-10000 Zagreb, Croatia \\ ${ }^{21}$ Department of Physics, University of California, Davis, CA 95616-8677, USA \\ ${ }^{22}$ Institute for Nuclear Research and Nuclear Energy, BG-1784 Sofia, Bulgaria \\ ${ }^{23}$ INAF/Osservatorio Astronomico and INFN, I-34143 Trieste, Italy \\ ${ }^{24}$ ICREA, E-08010 Barcelona, Spain \\ ${ }^{25}$ Dipartimento di Fisica, Università di Pisa and INFN Pisa, I-56126 Pisa, Italy \\ Received 2010 February 16; accepted 2011 January 13; published 2011 February 16
}

\section{ABSTRACT}

Between 2004 and 2009, a sample of 28 X-ray selected high- and intermediate-frequency peaked blazars with an $\mathrm{X}$-ray flux larger than $2 \mu \mathrm{Jy}$ at $1 \mathrm{keV}$ in the redshift range from 0.018 to 0.361 was observed with the MAGIC telescope at energies above $100 \mathrm{GeV}$. Seven among them were detected and the results of these observations are discussed elsewhere. Here we concentrate on the remaining 21 blazars which were not detected during this observation campaign and present the $3 \sigma(99.7 \%)$ confidence upper limits on their flux. The individual flux upper limits lie between $1.6 \%$ and $13.6 \%$ of the integral flux from the Crab Nebula. Applying a stacking method to the sample of non-detections with a total of $394.1 \mathrm{hr}$ exposure time, we find evidence for an excess with a cumulative significance of 4.9 standard deviations. It is not dominated by individual objects or flares, but increases linearly with the observation time as for a constant source with an integral flux level of $\sim 1.5 \%$ of that observed from the Crab Nebula above $150 \mathrm{GeV}$.

Key words: BL Lacertae objects: general - gamma rays: galaxies 


\section{INTRODUCTION}

MAGIC (Major Atmospheric Gamma-ray Imaging Cherenkov) is currently a system of two $17 \mathrm{~m}$ telescopes located atop the Roque de los Muchachos on the Canary Island of La Palma at $2200 \mathrm{~m}$ above sea level. The observations referred to in this study were obtained during the years 2004-2009 when MAGIC was still a single-dish telescope. Its $234 \mathrm{~m}^{2}$ tessellated parabolic mirror allows observations of Very High Energy (VHE) $\gamma$-rays between $\sim 50 \mathrm{GeV}$ and $10 \mathrm{TeV}$.

One key goal of the MAGIC telescope project is to determine the properties of extragalactic VHE sources, among which the high-frequency peaked BL Lacertae objects (HBLs) are the most numerous. Blazars are a subclass of radio-loud active galactic nuclei (AGNs) and belong to the most extreme and powerful objects in the universe. They are characterized by a non-thermal broadband continuum emission which is highly variable on timescales from years down to minutes (Albert et al. 2007; Aharonian et al. 2007a).

The spectral energy distribution (SED) of blazars is characterized by two bumps in a $v F_{v}$ representation. The first component peaks at energies between infrared and hard X-rays, and is assumed to originate from leptonic synchrotron radiation. The maximum of the second peak lies in the $\gamma$-ray energy regime. The origin of this peak can be explained by different and partially concurring models either relying on inverse Compton scattering of electrons (Maraschi et al. 1992; Dermer \& Schlickeiser 1993; Sikora et al. 1994) or proposing hadronic interactions inside the jet (Mannheim 1993; Muecke \& Protheroe 2001). In case the synchrotron peak occurs at energies above $\sim 10^{16.5} \mathrm{~Hz}$ (according to Nieppola et al. 2006), these blazars are called HBLs and for peak energies of $\sim 10^{14.5}-10^{16.5} \mathrm{~Hz}$, intermediate BL Lacertae objects (IBLs).

As of 2010 April, altogether 29 blazars were established as VHE sources (24 of them HBLs including M87 as a "misaligned" blazar), ${ }^{29}$ compared to 6 HBLs, when the MAGIC telescope began its regular observations in 2004 December. The sample presented here is comprised of $21 \mathrm{X}$-ray selected objects which were not detected in the VHE regime prior to the MAGIC observations. Nine of the objects were already observed between 2004 December and 2006 February and the upper limits of these observations are reported in Albert et al. (2008a). As there have been improvements within the MAGIC analysis, the data of these objects were re-analyzed and the new results are presented in this work. Since no significant detection was attained, upper limits on a $3 \sigma(99.7 \%)$ confidence level will be presented.

None of the observed sources showed any variability on diurnal timescales in the VHE regime. Assuming a positive detection in the case of a flaring state, the observations presented here provide a means of investigating the baseline emission of these objects. Therefore, a stacking method applied to the blazar sample can reveal such an emission below the sensitivity limit for each individual object. Together with VERITAS (Benbow 2009), this is the second stacking analysis which turns out to be successful in the VHE $\gamma$-ray regime. Former experiments like HEGRA failed to detect a significant signal in a stacking analysis

\footnotetext{
${ }^{26}$ Supported by INFN Padova.

${ }^{27}$ Now at Max-Planck-Institut für Kernphysik, D-69029 Heidelberg, Germany.

${ }^{28}$ Now at Centro de Investigaciones Energéticas, Medioambientales y Tecnológicas, Spain.

29 cf. http://wwwmagic.mppmu.mpg.de/ rwagner/sources/ for an up-to-date list.
}

due to their limited sensitivity (cf., for instance, Mannheim et al. 1996).

In Section 2, the selection criteria for the objects will be presented. The observations and the data analysis technique are described in Section 3. The analysis results are shown in Section 4. Finally, a discussion of the results and inherent implications can be found in Section 5 .

\section{BLAZAR SAMPLE}

We selected blazars from the compilations from Donato et al. (2001) and Costamante \& Ghisellini (2002). Additionally, some objects were chosen based on the synchrotron peak luminosity from Nieppola et al. (2006) and one from the sedentary survey by Giommi et al. (2005).

The main selection criteria are the measured X-ray flux at $1 \mathrm{keV}$ and the distance of the objects. According to Stecker et al. (1996), the synchrotron flux in the X-ray regime is connected to the flux in the VHE regime by

$$
v_{X} F_{X} \sim v_{\mathrm{TeV}} F_{\mathrm{TeV}}
$$

assuming comparable synchrotron and Compton peak luminosities. Therefore, objects with high X-ray fluxes are promising candidates for $\mathrm{TeV}$ emission. As the absorption of $\gamma$-rays within the extragalactic background light (EBL; see, e.g., Kneiske \& Dole 2010) is energy dependent, it is particularly important in the VHE regime to avoid strong attenuation of $\gamma$-rays by limiting the redshift range. According to Kneiske \& Dole (2010), at a redshift of $z=0.4$, the expected cutoff energy lies well above $200 \mathrm{GeV}$, allowing MAGIC to observe still with its highest sensitivity. Therefore, all objects with a maximum redshift $z=0.4$ were considered. The energy threshold of the observations increases with the zenith distance $\theta$. Accounting for this effect, the selection of sources with higher $\theta\left(30^{\circ}<\theta<45^{\circ}\right)$ during culmination should be limited to $z<0.15$. The increasing effect of EBL absorption should, however, imprint itself by a net steepening on the spectrum of the stacked excess.

All criteria are described in detail below. They have been chosen to enhance the probability to detect the sources, hence we selected objects with high fluxes and inverse Compton peaks as well as allowing for the lowest possible energies to be measured with MAGIC.

Compared to Albert et al. (2008a), the selection criteria have been extended. The reason is the enhancement of the sample by taking a wider redshift or zenith distance range into account and including sources whose fitted synchrotron peak flux is high enough even if they show a lower X-ray flux level at $1 \mathrm{keV}$. The sample is divided into four parts.

1. X-ray selected HBLs obtained from Donato et al. (2001) and Costamante \& Ghisellini (2002): (1) redshift $z<$ 0.4 , (2) X-ray flux $F_{x}(1 \mathrm{keV})>2 \mu \mathrm{Jy}$, and (3) zenith distance $\theta<30^{\circ}$ during culmination. Assuming the same luminosities at $1 \mathrm{keV}$ as at $200 \mathrm{GeV}$ (following the argumentation of Stecker et al. 1996), the X-ray flux $F_{x}(1 \mathrm{keV})=2 \mu \mathrm{Jy}$ corresponds to a $\gamma$-ray flux at $200 \mathrm{GeV}$ of $\sim 4.8 \times 10^{-12} \mathrm{erg} \mathrm{cm}^{-2} \mathrm{~s}^{-1}$. This criterion applies to 15 sources including 9 sources already observed during cycle 1 of regular MAGIC observations. The sources are listed in Table 1.

2. Two HBLs obtained from the same compilations taking a wider range in declination and a lower maximum of the 
Table 1

List of Targets

\begin{tabular}{|c|c|c|c|c|c|c|c|c|}
\hline Object & Season & $z$ & $\log \left(v_{p}\right)^{\mathrm{a}}$ & $F_{v_{p}}{ }^{\mathrm{b}}$ & $\begin{array}{c}F_{X}^{\mathrm{c}} \\
(\mu \mathrm{Jy})\end{array}$ & $\alpha_{X}{ }^{\mathrm{c}}$ & Cat. $^{\mathrm{d}}$ & $\begin{array}{c}\text { Sel. } \\
\text { Crit. }\end{array}$ \\
\hline 1ES $0033+595$ & 2006 Aug-2008 Jul & $0.086^{\mathrm{f}}$ & 18.9 & 2.0 & 5.66 & $\ldots$ & $\mathrm{C}^{\dagger}, \mathrm{N}$ & II \\
\hline 1ES $0120+340$ & 2005 Aug-Sep & 0.272 & 18.3 & 2.5 & 4.34 & 1.93 & $\mathrm{C}, \mathrm{D}^{\dagger}, \mathrm{G}, \mathrm{N}$ & I \\
\hline 1ES $0229+200^{\mathrm{g}}$ & 2006 Aug-Nov & 0.140 & 19.5 & 1.6 & 2.88 & $\ldots$ & $\mathrm{C}^{\dagger}, \mathrm{N}$ & $\mathrm{I}, \mathrm{II}$ \\
\hline RX J0319.8+1845g & 2004 Dec-2006 Jan & 0.190 & 17.0 & 0.4 & 1.76 & 2.07 & $\mathrm{D}^{\dagger}, \mathrm{G}, \mathrm{N}$ & I \\
\hline 1ES $0323+022$ & 2005 Sep-Dec & 0.147 & 19.9 & 6.3 & 3.24 & 2.46 & $\mathrm{C}, \mathrm{D}^{\dagger}, \mathrm{G}, \mathrm{N}$ & $\mathrm{I}, \mathrm{II}$ \\
\hline $1 \mathrm{ES} 0414+009^{\mathrm{g}}$ & 2005 Dec-2006 Jan & 0.287 & 20.7 & 10.0 & 5.00 & 2.49 & $\mathrm{C}, \mathrm{D}^{\dagger}, \mathrm{G}, \mathrm{N}$ & I \\
\hline 1RXS J044127.8+150455 & 2007 Oct-Dec & 0.109 & $\ldots$ & $\ldots$ & 4.74 & 2.10 & $\mathrm{G}^{\dagger}$ & IV \\
\hline $1 \mathrm{ES} 0647+250$ & 2008 Feb-Mar & $0.203^{\mathrm{f}}$ & 18.3 & 3.2 & 6.01 & 2.47 & $\mathrm{C}^{\dagger}, \mathrm{D}, \mathrm{N}$ & I \\
\hline $1 \mathrm{ES} 0806+524^{\mathrm{g}}$ & 2005 Oct-Dec & 0.138 & 16.6 & 1.6 & 4.91 & 2.93 & $\mathrm{C}, \mathrm{D}^{\dagger}, \mathrm{N}$ & $\mathrm{I}, \mathrm{II}$ \\
\hline 1ES $0927+500$ & 2005 Dec-2006 Feb & 0.188 & 21.1 & 5.0 & 4.00 & 1.88 & $\mathrm{D}^{\dagger}, \mathrm{G}, \mathrm{N}$ & I \\
\hline 1ES $1011+496^{\mathrm{g}}$ & 2006 Mar-Apr & 0.212 & 16.7 & 1.3 & 2.15 & 2.49 & $\mathrm{C}, \mathrm{D}^{\dagger}, \mathrm{N}$ & I \\
\hline 1ES $1028+511$ & 2007 Mar-2008 Feb & 0.361 & 18.6 & 1.3 & 4.42 & 2.50 & $\mathrm{C}, \mathrm{D}^{\dagger}, \mathrm{G}, \mathrm{N}$ & I \\
\hline RGB J1117+202 & 2007 Jan-2008 Mar & 0.140 & $\ldots$ & $\ldots$ & 6.93 & 1.90 & $\mathrm{C}^{\dagger}, \mathrm{D}, \mathrm{G}$ & I,II \\
\hline RX J1136.5+6737 & $2007 \mathrm{Feb}$ & 0.135 & 17.6 & 1.3 & 3.17 & 2.39 & $\mathrm{C}, \mathrm{D}^{\dagger}, \mathrm{G}, \mathrm{N}$ & II \\
\hline B2 $1215+30$ & 2007 Mar-2008 Mar & 0.237 & 15.6 & 1.3 & 1.59 & 2.65 & $\mathrm{C}, \mathrm{D}, \mathrm{N}^{\dagger \mathrm{h}}$ & III \\
\hline 2 E $1415.6+2557$ & 2005 Apr-2008 Apr & 0.237 & 19.2 & 3.2 & 3.26 & 2.25 & $\mathrm{C}, \mathrm{D}^{\dagger}, \mathrm{G}, \mathrm{N}$ & I \\
\hline PKS $1424+240^{9}$ & 2006 May-2007 Feb & $0.160^{\mathrm{f}}$ & 15.7 & 1.0 & 1.37 & 2.98 & $\mathrm{D}, \mathrm{N}^{\dagger \mathrm{h}}$ & III \\
\hline RX J1725.0+1152 & 2005 Apr-2009 Apr & $0.018^{\mathrm{f}}$ & 15.8 & 2.0 & 3.60 & 2.65 & $\mathrm{C}, \mathrm{D}^{\dagger}, \mathrm{N}$ & $\mathrm{I}, \mathrm{II}$ \\
\hline 1ES $1727+502$ & 2006 May-2007 May & 0.055 & 17.4 & 1.3 & 3.36 & 2.61 & $\mathrm{C}, \mathrm{D}^{\dagger}, \mathrm{N}$ & $\mathrm{I}, \mathrm{II}$ \\
\hline 1ES $1741+196$ & $2006 \mathrm{Jul}-2007$ Apr & 0.083 & 17.9 & 1.0 & $1.92^{\mathrm{i}}$ & 2.04 & $\mathrm{C}, \mathrm{D}^{\dagger}, \mathrm{N}$ & $\mathrm{I}, \mathrm{II}$ \\
\hline B3 $2247+381$ & 2006 Aug-Sep & 0.119 & 15.6 & 1.0 & 0.60 & 2.51 & $\mathrm{D}, \mathrm{N}^{\dagger \mathrm{h}}$ & III \\
\hline
\end{tabular}

Notes. List of objects in the sample of X-ray selected blazars with their observation time windows, redshifts, and X-ray measurements.

${ }^{a}$ Fitted peak frequency from Nieppola et al. (2006) in units of $\log (\mathrm{Hz})$.

${ }^{b}$ Flux at peak frequency extracted from Nieppola et al. (2006) in units of $10^{-11} \mathrm{erg} \mathrm{cm}^{-2} \mathrm{~s}^{-1}$.

${ }^{\mathrm{c}}$ Flux and photon spectral index at $1 \mathrm{keV}$.

d Compilation where the object appears (C: Costamante \& Ghisellini 2002; D: Donato et al. 2001; N: Nieppola et al. 2006; G: Giommi et al. 2005). The catalog from which the object was selected is marked with a dagger.

${ }^{\mathrm{e}}$ Selection criteria which are met by the object.

f Tentative redshift.

g Known VHE blazar (as of 2010 April) due to a detection after the MAGIC observation period.

${ }^{\mathrm{h}}$ The objects chosen from Nieppola et al. (2006) are also listed in Donato et al. (2001), but with an X-ray flux lower than $2 \mu \mathrm{Jy}$.

${ }^{i}$ Mean X-ray flux of multiple measurement in Donato et al. (2001) below $2 \mu \mathrm{Jy}$.

redshift into account: 1ES $0033+59.5$ and RXS J1136.5+ 6737. Selection criteria: (1) redshift $z<0.15$, (2) X-ray flux $F_{x}(1 \mathrm{keV})>2 \mu \mathrm{Jy}$, and (3) $\theta<45^{\circ}$ during culmination.

3. Intermediate BL Lacertae objects taken from Nieppola et al. (2006) with high peak luminosities at the synchrotron peak. Selection criteria: (1) redshift $z<0.4$, (2) synchrotron peak frequency $v_{\text {peak }}>2 \times 10^{15} \mathrm{~Hz}$, (3) flux at the peak $F_{v_{\text {peak }}}>10^{-11} \mathrm{erg} \mathrm{cm}^{-2} \mathrm{~s}^{-1}$, and (4) zenith angle $\theta<30^{\circ}$ during culmination. This is valid for three sources: B2 $1215+30$, PKS $1424+240$, and B3 $2247+381$. All of them can also be found in Donato et al. (2001) but with an $\mathrm{X}$-ray flux at $1 \mathrm{keV}$ below $2 \mu \mathrm{Jy}$. B2 $1215+30$ is listed there as a low-frequency peaked BL Lacertae object. In return, it is included in the $\mathrm{TeV}$ candidate list in Costamante \& Ghisellini (2002).

4. One HBL from the sedentary survey (Giommi et al. 2005) with the same selection criteria as applied for point one of the sample: 1RXS J044127.8+150455.

As several other blazars fulfilling these selection criteria were already detected with MAGIC or other VHE instruments, a post-priori selection was done using only the objects which were not yet detected in the VHE regime in advance of the MAGIC observations leaving 21 objects as discussed herein. All blazars in the MAGIC AGN observation program that fulfill these selection criteria either have been detected (or were known in advance) or listed here as non-detections.
Table 1 lists all sources in the sample with relevant parameters. In the case of multiple flux or spectral slope measurements, the mean value is displayed.

\section{OBSERVATIONS AND DATA ANALYSIS TECHNIQUE}

The observations presented here were carried out between 2004 December and 2009 April with a total amount of observation time of $490.0 \mathrm{hr}$. After quality selection (removing low-quality data runs from the analysis), $394.1 \mathrm{hr}$ were used for the analysis or $18.8 \mathrm{hr}$ per source on average. The main reason for discarding data from the analysis is a low event rate after image cleaning which is primarily influenced by the weather conditions.

Most of the data were taken in wobble mode. In this mode, the pointing position of the telescope is displaced by 0.4 from the source position. In order to get a well-balanced coverage inside the camera, the wobble position is changed regularly to the opposite (with respect to the source position). Signal and background events are then determined from the same shower images with respect to the source position and to three symmetric OFF positions, respectively, all at the same distance to the camera center. Part of the data of RX J0319.8+1845, 2E 1415.6+2557, and RX J1725.0+1152 were taken in the ON mode where the pointing position of the telescope is centered on the object in the sky. For these observations dedicated OFF observations have been used for the background estimation. 
The data were processed with the software package MARS (Bretz 2005) using an automated analysis pipeline. Details can be found in Bretz \& Wagner (2003), Bretz \& Dorner (2008), and Albert et al. (2008b). Furthermore, the arrival time information of neighboring pixels was taken into account (Aliu et al. 2009).

For the separation of signal and background events, dynamic cuts on the distribution of image parameters are applied. The image parameters are moments up to third order in the light distribution of the shower images (Hillas 1985). The background suppression is done by means of a parabolic cut in AREA (Riegel et al. 2005) and a cut in $\vartheta^{2}$. The latter parameter is the squared angular distance between the source position and the reconstructed shower origin determined with a refined DISP method (Lessard et al. 2001) taking into account the timing information of the showers. The $\vartheta^{2}$ cut used in this analysis is $\vartheta^{2}<0.0196$ which is a somewhat smaller value than usually used for the Crab Nebula, but provides a better background rejection for weak point sources. The chosen value for $\vartheta^{2}$ corresponds to a signal region in the camera plane with a diameter of 2.8 camera pixels. The optical point spread function of the MAGIC telescope during the campaign was smaller than $16.0 \mathrm{~mm}$ corresponding to a diameter of 1.1 pixels, well within this area. A large sample of objects spanning a long time of observations has to be treated with a robust analysis. The usage of dynamic cuts provides such an analysis on the expense of sensitivity (cf. Section 4.1).

The statistical significance for any excess is calculated from the $\vartheta^{2}$ distribution of signal and background events making use of Equation (17) in Li \& Ma (1983).

Concerning the stacking method as described in Sections 4.4 and 4.5 , the $\vartheta^{2}$ distributions have been summed up to retrieve the stacked $\vartheta^{2}$ signal plot. The differential energy spectrum is then calculated from all excess events using average values for the effective collection area and a Monte Carlo correction factor (spillover correction), each weighted with the exposure time $t_{\text {exp }}$. The same method has been applied to a data set of the Crab Nebula (cf. Section 4.1) demonstrating its feasibility.

\section{RESULTS OF THE MAGIC OBSERVATIONS}

During the observation campaign, no significant detection of any individual object could be achieved. The results can be found in Table 3 . None of the objects showed flaring activity in the VHE band on a significant level on diurnal timescales within the observation time windows. Flaring activity is defined here as an offset of 3 standard deviations from the mean measured $\gamma$ rate for each object. However, flux variations by a factor of three would still prevent an individual object of the sample from being detected with high significance. In this section, we present the upper limits obtained for all 21 objects.

Three of the objects were partially observed during an optical high state within a target of opportunity campaign. The trigger criterion was an increase in the optical flux of the core of more than $50 \%$. The objects are 1ES $0033+595$, RGB J1117+202, and B2 $1215+30$. Significant activity or variability in the VHE $\gamma$-ray regime could not be detected.

\subsection{Crab Nebula Observations}

For a comparative analysis, a sample of the Crab Nebula data has been used spanning a time range from 2005 October to 2008 January. Three data sets have been chosen to account for the three different hardware conditions during the blazar observations: a $300 \mathrm{MHz}$ readout system without and with optical splitters and a $2 \mathrm{GHz}$ readout system, later on referred to as $300 \mathrm{MHz}, 300 \mathrm{MHz}_{\mathrm{OS}}$, and $2 \mathrm{GHz}$ systems, respectively. The $\theta$ distribution of the subsamples has been matched to that of the blazar sample; the overall observation time after quality selection is $t_{\exp }=19.2 \mathrm{hr}$. The individual values as well as the combined result can be found in Table 2 . The energy spectrum can be fitted with a log parabola (according to Equation (2) in Albert et al. 2008c) accounting for the flattening of the spectrum toward the inverse Compton peak

$$
\frac{d N}{d E}=f_{0} \cdot\left(\frac{E}{300 \mathrm{GeV}}\right)^{\left[a+b \log _{10}(E / 300 \mathrm{GeV})\right]}
$$

with $f_{0}=(5.37 \pm 0.11) \times 10^{-10} \mathrm{TeV}^{-1} \mathrm{~cm}^{-2} \mathrm{~s}^{-1}, a=-2.20 \pm$ 0.05 , and $b=-0.11 \pm 0.03$. The $\vartheta^{2}$ distribution and the energy spectrum have been calculated in the very same way as for the blazar sample by stacking the three individual Crab Nebula samples. The integral flux above $150 \mathrm{GeV}$ is determined to be $F_{>150 \mathrm{GeV}}=(2.81 \pm 0.05) \times 10^{-10} \mathrm{~cm}^{-2} \mathrm{~s}^{-1}$. It will be used for comparison with the integral upper limits derived from the blazars. Figure 1 displays the energy spectrum of the stacked excess of the Crab Nebula in comparison to the published spectrum. The integral flux above $150 \mathrm{GeV}$ amounts to $91 \%$ of that determined in Albert et al. (2008c). A comparison to previous measurements of experiments such as HEGRA, HESS, or Whipple is difficult because of the higher energy threshold of these measurements (above $400 \mathrm{GeV}$ ). Due to the hardening of the Crab spectrum toward the peak below $100 \mathrm{GeV}$, a simple extrapolation of the power-law spectra found there overestimates the flux at $150 \mathrm{GeV}$ leading to integral flux ratios of $\sim 70 \%-80 \%$ above $150 \mathrm{GeV}$ (cf. Aharonian et al. 2000, 2006; Grube 2007).

The standard MAGIC integral sensitivity is $\sim 1.6 \%$ of the Crab Nebula flux above $280 \mathrm{GeV}$ for detecting a signal with $5 \sigma$ in $50 \mathrm{hr}$ (Aliu et al. 2009). Including lower energies in the integral sensitivity determination, the value increases. The analysis presented in this work has an integral sensitivity above $150 \mathrm{GeV}$ of $3.8 \%$ of the Crab Nebula flux. This is mainly due to the long-term characteristics of the observations, because the analysis is aimed at a robust and conservative treatment of the data; in addition, data before the installation of the $2 \mathrm{GHz}$ system are also considered, where the standard MAGIC sensitivity above $280 \mathrm{GeV}$ is also less with $\sim 1.9 \%$ of the Crab Nebula flux.

\subsection{Upper Limits}

The upper limits (U.L.) on the excess rates are calculated on a confidence level of $3 \sigma(99.7 \%)$ using the method from Rolke et al. (2005). Integral flux upper limits above a given energy are then calculated from them. The integral flux for each source is given above the energy threshold of the analysis, which is defined as the maximum of the differential distribution $d N / d E$ versus $E$ of simulated $\gamma$-showers surviving all cuts. The integral fluxes are also compared to the integral flux of the Crab Nebula above the individual thresholds.

The energy estimation for each source was done based on Monte Carlo simulated $\gamma$ events following a power-law distribution with $\Gamma=-3.0$ for a power law $d N / d E \propto E^{\Gamma}$. This was done in order to fit better the average spectral slope for the blazars in the VHE regime. For the integral upper limit calculation, the same input spectrum $(\Gamma=-3.0)$ was used. The resulting upper limits vary between $1.6 \%$ and $13.6 \%$ of the Crab Nebula flux above the individual energy threshold. The energy thresholds lie between $120 \mathrm{GeV}$ and $230 \mathrm{GeV}$ due to 


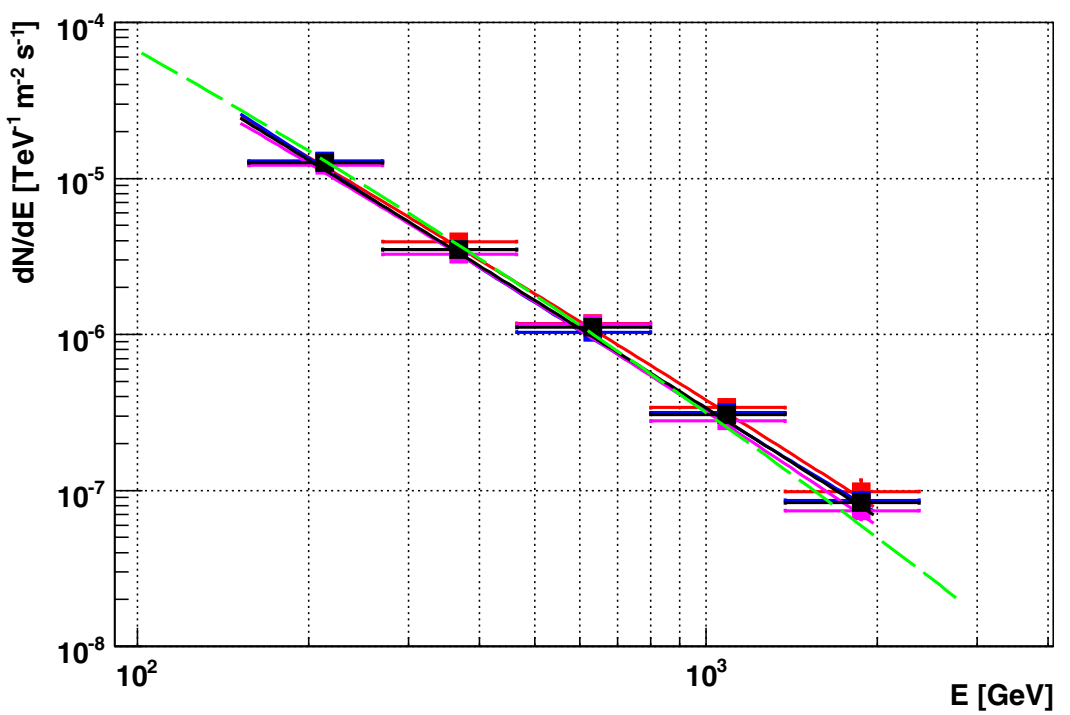

Figure 1. Observations of three data sets of the Crab Nebula between 2005 October and 2008 January. The red, blue, and magenta colored data represent the data sets of the $300 \mathrm{MHz}, 300 \mathrm{MHz}_{\mathrm{OS}}$, and the $2 \mathrm{GHz}$ systems, respectively. The black curve shows the combined energy spectrum obtained with the stacking method. For comparison the published spectrum from Albert et al. (2008c) is plotted as dashed green line. Note that the vertical error bars are hidden by the marks.

Table 2

Observations of the Crab Nebula

\begin{tabular}{|c|c|c|c|c|c|c|c|}
\hline Season & $\begin{array}{l}\text { FADC } \\
\text { System }\end{array}$ & $\begin{array}{l}t_{\text {exp }} \\
(\mathrm{hr})\end{array}$ & $\begin{array}{c}\theta \\
\left({ }^{\circ}\right)\end{array}$ & $\begin{array}{l}\text { Excess } \\
\text { Events }\end{array}$ & $\begin{array}{l}\text { Background } \\
\text { Events }\end{array}$ & $\begin{array}{c}\text { Significance } \\
\sigma\end{array}$ & $\begin{array}{c}E_{\text {thr }} \\
(\mathrm{GeV})\end{array}$ \\
\hline 2005 Oct-2006 Mar & $300 \mathrm{MHz}$ & 3.8 & $6-37$ & 967 & 209 & 36.0 & 165 \\
\hline 2006 Sep-2007 Jan & $300 \mathrm{MHz}_{\mathrm{OS}}$ & 8.1 & $7-43$ & 2086 & 523 & 51.0 & 165 \\
\hline 2007 Feb-2008 Jan & $2 \mathrm{GHz}$ & 7.3 & $8-30$ & 2133 & 455 & 53.5 & 165 \\
\hline Combined & $\ldots$ & 19.1 & $6-43$ & 5188 & 1188 & 82.2 & 165 \\
\hline
\end{tabular}

Notes. Observations of the Crab Nebula used for a performance test of the stacking method and comparison to the flux upper limits of the blazars. The final spectrum (cf. Equation (2)) is obtained as a combination of all the subsamples.

differences in the $\theta$ distributions of the individual data samples. The Monte Carlo simulations have been chosen to match exactly the $\theta$ distribution of each data sample. The results of the spectral analysis can be found in Table 3, too.

Discovery of VHE $\gamma$-rays from RX J0319.8+1845 and 1ES $0806+524$ has recently been reported by the VERITAS collaboration (Acciari et al. 2009; Ong \& Fortin 2009), as well as from PKS $1424+240$ which was confirmed by the MAGIC collaboration in a campaign independent of the observations presented here (Acciari et al. 2010; Teshima 2009). The measured VHE flux for the latter source was significantly higher than in previous observations with the MAGIC telescope. 1ES 0229+200 and 1ES 0414+009 have been detected by the HESS telescope array in 2006 (Aharonian et al. 2007b) and 2009 (Hofmann \& Fegan 2009), respectively. However, since the observations presented here were each performed in advance of the detections mentioned above, the inclusion of these sources in the stacking method is justified. The later detections show that the X-ray selection of possible targets is a reasonable approach.

In order to compare the measured integral fluxes with the upper limits presented here they are extrapolated to the individual energy thresholds as reported in Table 3. In all cases except for PKS 1424+240, the upper limits are compatible with the extrapolated reported integral fluxes.

\subsection{Significance Distribution}

Taking a look at the calculated significances of the blazar sample, it is evident that most of the individual objects show positive values. Plotting the distribution of the significances, the mean value is not located at 0 as expected for sky regions where no $\gamma$-rays are expected to originate.

In Figure 2, the significance distribution for the blazar sample is shown together with the result of a cross-check as described below. As the number of individual samples is different for both distributions they have been normalized to one. The blazar sample distribution has a mean value of $1.23 \pm 1.17$, while the cross-check sample has $-0.08 \pm 0.85$. This result can be expected due to the fact that our sample is biased by the selection toward potential VHE $\gamma$-ray emitters.

In order to test if the positive signal in the blazar sample originates from a systematic effect of the observations or analysis chain, we also cross-checked this result with data sets obtained as OFF pointings associated with different ON source observations not treated in this paper. These data sets were taken under similar conditions as the blazars covering the whole range of $\theta$ of the blazar sample and processed with the very same analysis chain. The OFF observations were analyzed in wobble mode with respect to two fake source positions in the camera displaced by 0.4 from the camera center. Table 4 gives a list of these observations and results. Although the fit parameters of Gaussian fits to both distributions do not permit any conclusive statement, a Kolmogorov-Smirnov test of the compatibility of the blazar with the cross-check sample gives a probability of $1.56 \%$. For the Gaussian distributions the test returns $3.42 \%$ and $77.03 \%$ for the compatibility of the blazar and the crosscheck sample with the standard Gaussian, respectively. The 


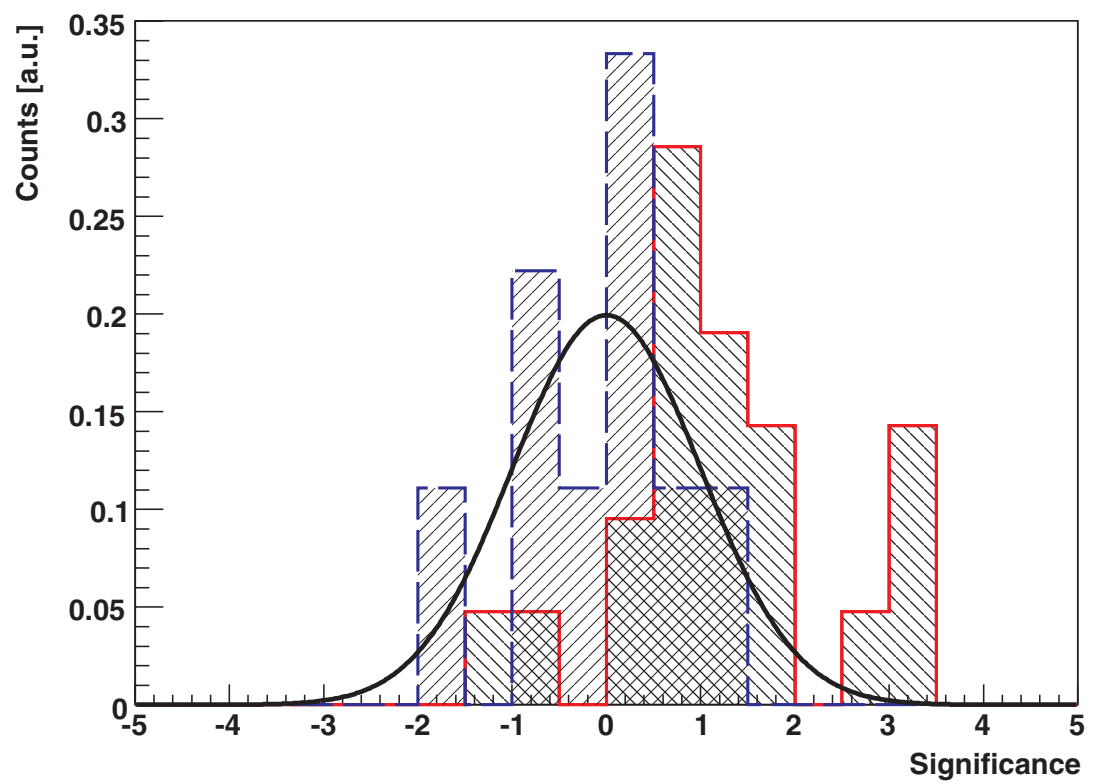

Figure 2. Significance distributions of the blazar (red, hatched up left to low right) and the cross-check sample (blue, hatched low left to up right). The different distributions are normalized to one, so the vertical axis gives the percentage of the whole blazar or cross-check sample, respectively. The blazar sample distribution has a mean value of $1.23 \pm 1.17$ and the cross-check sample, $-0.08 \pm 0.85$. For comparison a Gaussian with mean value 0 and standard deviation 1 is plotted as a black curve.

Table 3

Results of the Analysis

\begin{tabular}{|c|c|c|c|c|c|c|c|c|c|}
\hline Object & $\begin{array}{l}t_{\exp } \\
(\mathrm{hr})\end{array}$ & $\begin{array}{c}\theta \\
\left({ }^{\circ}\right)\end{array}$ & $\begin{array}{l}\text { Excess } \\
\text { Events }\end{array}$ & $\begin{array}{l}\text { Background } \\
\text { Events }\end{array}$ & Scale & $\begin{array}{c}\text { Significance } \\
(\sigma)\end{array}$ & $\begin{array}{c}E_{\mathrm{thr}}{ }^{\mathrm{a}} \\
(\mathrm{GeV})\end{array}$ & $\begin{array}{l}\text { U.L. } \\
\text { (c.u. })^{\text {b }}\end{array}$ & $\begin{array}{l}\text { U.L. } \\
\text { (f.u. })^{\mathrm{c}}\end{array}$ \\
\hline 1ES $0033+595$ & 5.2 & $31-41$ & 60.0 & 331.0 & 0.33 & 2.8 & 170 & 9.7 & 2.4 \\
\hline $1 \mathrm{ES} 0120+340$ & 10.7 & $6-18$ & 20.7 & 437.3 & 0.33 & 0.9 & 120 & 8.2 & 3.1 \\
\hline $1 \mathrm{ES} 0229+200$ & 8.0 & $8-37$ & 55.0 & 572.0 & 0.33 & 2.0 & 120 & 13.6 & 5.1 \\
\hline RX J0319.8+1845 & 11.2 & $10-31$ & -23.4 & 631.4 & 0.59 & -0.7 & 120 & 1.6 & 0.6 \\
\hline 1ES $0323+022$ & 11.4 & $26-46$ & -45.3 & 751.3 & 0.33 & -1.5 & 170 & 6.9 & 1.7 \\
\hline 1ES $0414+009$ & 18.2 & $28-36$ & 71.3 & 1020.7 & 0.33 & 1.9 & 170 & 7.7 & 1.9 \\
\hline 1RXS J044127.8+150455 & 26.9 & $13-36$ & 18.3 & 1825.7 & 0.33 & 0.4 & 120 & 3.2 & 1.2 \\
\hline $1 \mathrm{ES} 0647+250$ & 29.2 & $3-32$ & 64.3 & 1797.7 & 0.33 & 1.3 & 120 & 4.3 & 1.6 \\
\hline 1ES 0806+524 & 17.5 & $24-36$ & 17.0 & 752.0 & 0.33 & 0.5 & 140 & 7.2 & 2.2 \\
\hline $1 \mathrm{ES} 0927+500$ & 16.7 & $21-26$ & 28.3 & 702.7 & 0.33 & 0.9 & 140 & 5.6 & 1.7 \\
\hline 1ES $1011+496$ & 14.5 & $21-29$ & 89.0 & 590.0 & 0.33 & 3.1 & 140 & 6.9 & 2.1 \\
\hline 1ES $1028+511$ & 37.1 & $22-36$ & 65.7 & 2312.3 & 0.33 & 1.2 & 140 & 3.3 & 1.0 \\
\hline RGB J1117+202 & 14.9 & $8-38$ & 25.7 & 804.3 & 0.33 & 0.8 & 120 & 5.3 & 2.0 \\
\hline RX J1136.5+6737 & 14.8 & $39-46$ & 22.7 & 954.3 & 0.33 & 0.6 & 230 & 5.7 & 0.9 \\
\hline B2 $1215+30$ & 16.1 & $1-41$ & 119.0 & 995.0 & 0.33 & 3.2 & 120 & 9.3 & 3.5 \\
\hline 2 E $1415.6+2557$ & 57.4 & $3-36$ & 7.6 & 3805.4 & 0.54 & 0.1 & 120 & 3.5 & 1.3 \\
\hline PKS $1424+240$ & 20.0 & $5-36$ & 51.7 & 1210.3 & 0.33 & 1.3 & 120 & 8.2 & 3.1 \\
\hline RX J1725.0+1152 & 32.0 & $17-35$ & 70.0 & 1859.0 & 0.38 & 1.4 & 140 & 4.2 & 1.3 \\
\hline $1 \mathrm{ES} 1727+502$ & 6.1 & $21-36$ & 31.0 & 302.0 & 0.33 & 1.5 & 140 & 11.8 & 3.6 \\
\hline 1ES $1741+196$ & 11.8 & $9-40$ & 98.7 & 731.3 & 0.33 & 3.1 & 120 & 9.6 & 3.6 \\
\hline B3 $2247+381$ & 8.3 & $10-36$ & 21.7 & 490.3 & 0.33 & 0.8 & 140 & 5.2 & 1.6 \\
\hline
\end{tabular}

Notes. Results of the analysis. The upper limits span a range of 1.6\%-13.6\% of the Crab Nebula flux above the corresponding energy threshold.

${ }^{a}$ Peak response energy for a power-law spectrum $E^{\Gamma}$ with $\Gamma=-3.0$.

${ }^{\mathrm{b}}$ Integral flux above $E_{\mathrm{thr}}$ given in units of the flux of the Crab Nebula (crab units, c.u.).

${ }^{\mathrm{c}}$ Integral flux above $E_{\mathrm{thr}}$ given in flux units (f.u.) $=10^{-11} \mathrm{~cm}^{-2} \mathrm{~s}^{-1}$.

cross-check sample is $\sim 7$ times smaller than the blazar sample, and thus systematic effects in the analysis can only largely be ruled out as a possible explanation for the shift in the blazar distribution.

\subsection{Stacking Analysis}

Even if none of the sources was detected in a single observation, a cumulative signal search seems promising. For this reason the $\vartheta^{2}$ plots of the individual analyses have been stacked producing one plot for the whole set containing $394.1 \mathrm{hr}$ of data (cf. Section 3). Figure 3 shows the result, a significance of 4.9 standard deviations with 870 excess and 22,876 background events. About $30 \%$ of the stacked excess comes from blazars now known as VHE $\gamma$-ray emitters. Without these sources the stacked excess amounts to 608 excess events with a significance of $3.8 \sigma$ indicating that there are other emitters contained 


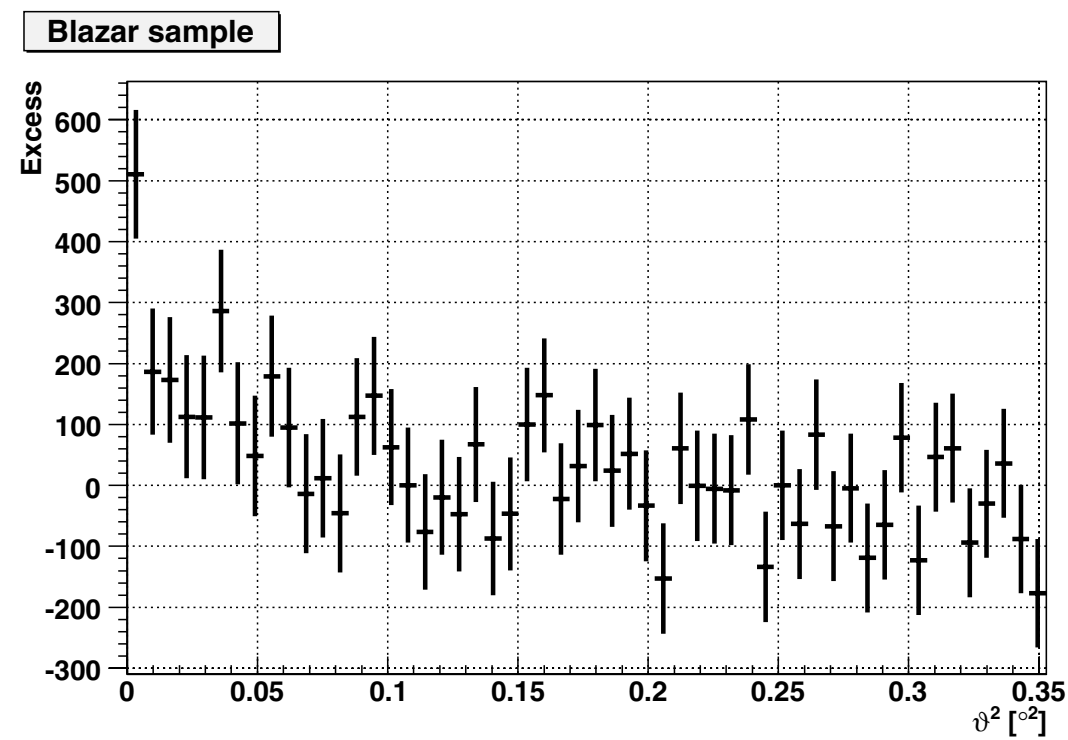

\section{Cross-check sample}

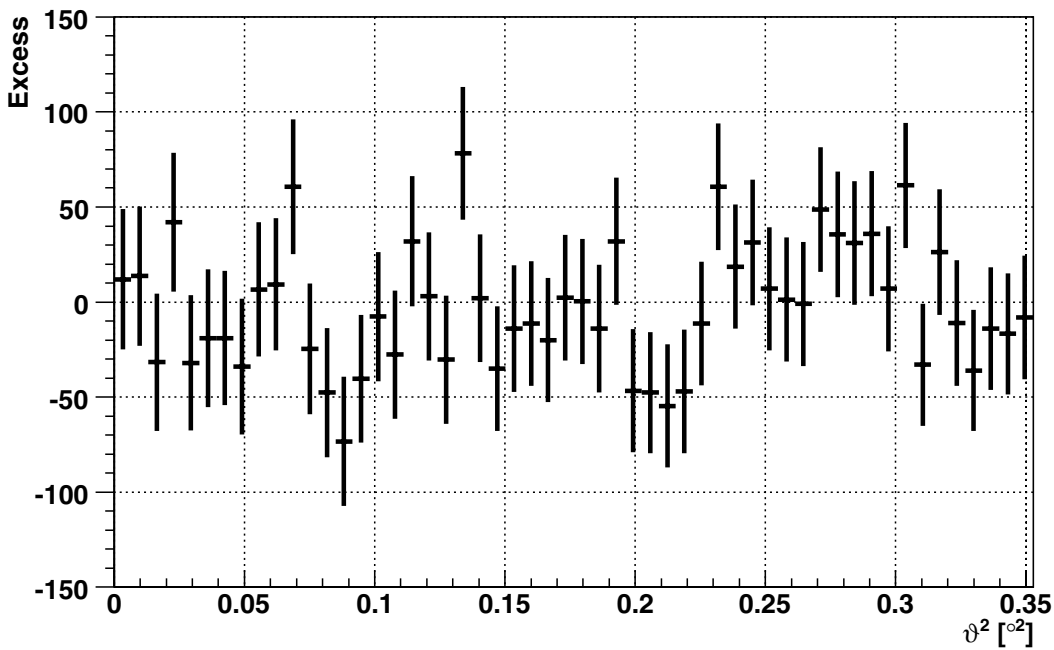

Figure 3. $\vartheta^{2}$ distribution of excess events for the stacked blazar sample (top) and the cross-check sample (bottom). The blazar sample shows a clear extension at low values with a significance of 4.9 standard deviations.

Table 4

Data Samples for Cross-check

\begin{tabular}{llrrrrr}
\hline \hline Sample & \multicolumn{1}{c}{ Season } & $\begin{array}{c}t_{\exp } \\
(\mathrm{hr})\end{array}$ & $\begin{array}{c}c \\
\left({ }^{\circ}\right)\end{array}$ & $\begin{array}{r}\text { Excess } \\
\text { Events }\end{array}$ & $\begin{array}{r}\text { Background } \\
\text { Events }\end{array}$ & $\begin{array}{r}\text { Significance } \\
(\sigma)\end{array}$ \\
\hline 1 & 2006 Jun-Jul & 5.4 & $34-43$ & -1.3 & 335.3 & -0.1 \\
2 & 2006 Jul & 3.1 & $6-29$ & 4.3 & 107.7 & 0.4 \\
3 & 2006 Nov & 1.9 & $37-47$ & 19.0 & 255.0 & 1.0 \\
4 & 2007 Jan & 3.3 & $49-56$ & -24.7 & 149.7 & -1.8 \\
5 & 2007 Apr & 2.8 & $11-27$ & -9.7 & 139.7 & -0.7 \\
6 & 2007 May & 1.3 & $28-37$ & 2.0 & 76.0 & 0.2 \\
7 & 2007 May & 7.3 & $29-36$ & -20.7 & 356.7 & -1.0 \\
8 & 2008 Jan-Aug & 17.9 & $22-38$ & 7.0 & 1041.0 & 0.2 \\
9 & 2008 Feb-Apr & 9.3 & $22-26$ & 18.0 & 548.0 & 0.7 \\
\hline
\end{tabular}

Notes. Data samples used for the cross-check analysis. They were chosen to give a good coverage of the $\theta$ distributions and the different night sky background conditions of the blazar sample.

in the sample. Figure 4 underlines this finding. As expected, the stacked $\vartheta^{2}$ plot of the cross-check analysis containing no $\gamma$-signal gives a significance of -0.1 with -6 excess and 3009 background events; the result is shown in Figure 3 as well.

\subsection{Energy Spectrum}

From the combined excess a differential energy spectrum can be calculated. The differential energy spectrum $d F / d E$ for one source is calculated binwise by dividing the product of the number of excess events $N_{\mathrm{exc}, \mathrm{i}}$ and the spillover factor $a_{i}$ by the product of effective collection area $A_{\text {eff,i }}$ and exposure time $t_{\text {exp }}$. In order to derive an energy spectrum of the stacked excess, the mean values of $a_{i}$ and $A_{\text {eff,i }}$ weighted with the observation time have to be taken:

$$
\begin{aligned}
\left\langle a_{i}\right\rangle & =\frac{\sum_{n} a_{i, n} \cdot t_{\text {exp }, \mathrm{n}}}{\sum_{n} t_{\text {exp }, \mathrm{n}}} \\
\left\langle A_{\mathrm{eff}, \mathrm{i}}\right\rangle & =\frac{\sum_{n} A_{\text {eff }, \mathrm{i}, \mathrm{n}} \cdot t_{\mathrm{exp}, \mathrm{n}}}{\sum_{n} t_{\mathrm{exp}, \mathrm{n}}}
\end{aligned}
$$

with $n$ being the number of objects to be stacked and the energy bin $i$. The differential quotient $d N_{i} / d E$ for each bin can then be calculated as

$$
\frac{d N_{i}}{d E}=\frac{\sum_{n} N_{\mathrm{exc}, \mathrm{i}, \mathrm{n}} \cdot\left\langle a_{i}\right\rangle}{\sum_{n} t_{\mathrm{exp}, \mathrm{n}} \cdot\left\langle A_{\mathrm{eff}, \mathrm{i}}\right\rangle \cdot \Delta E_{i}}
$$




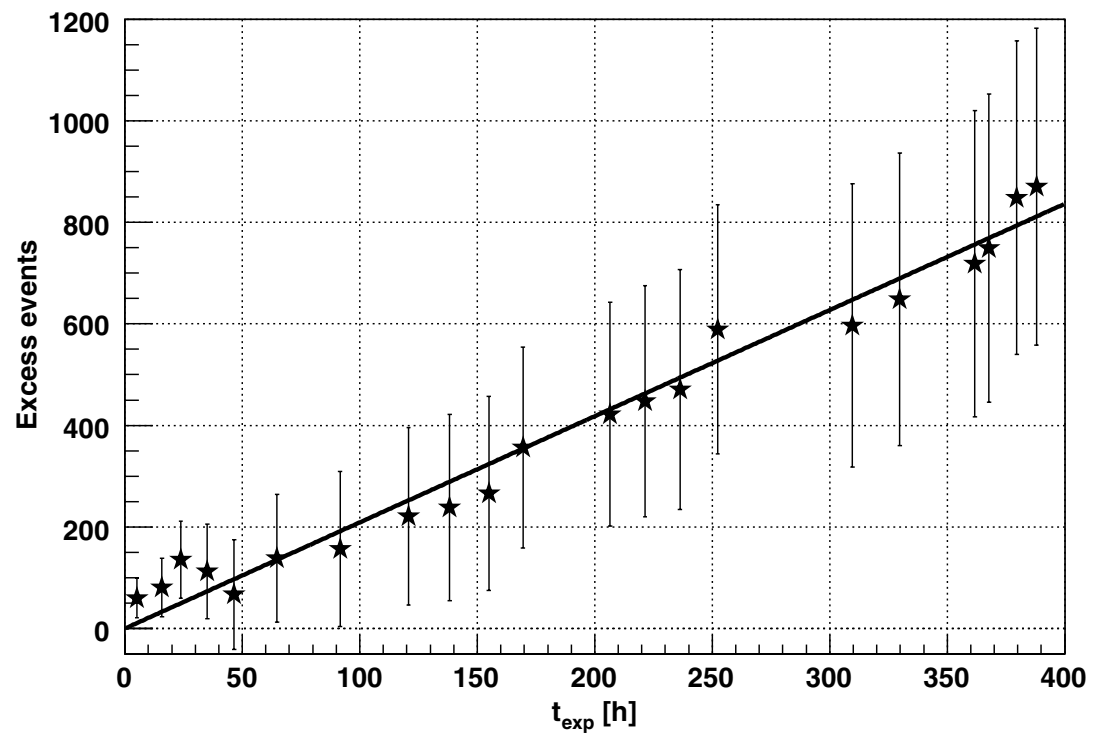

Figure 4. Excess events of the individual blazars vs. the overall exposure time. On average, each blazar contributes $2.1 \pm 0.3$ excess events per hour.

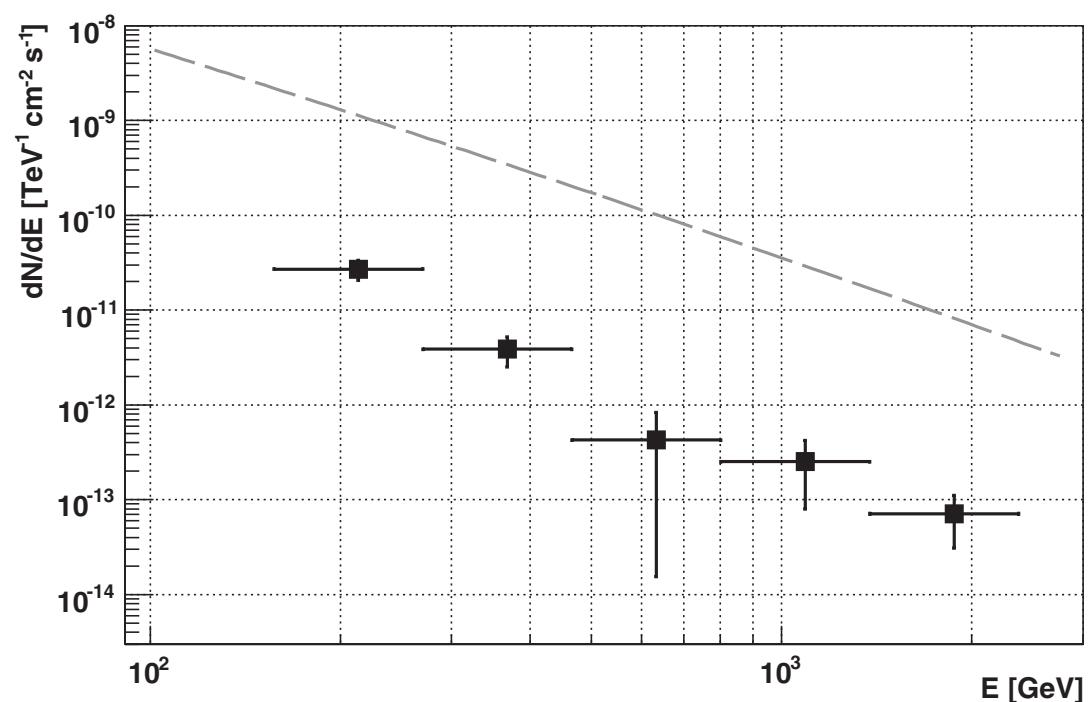

Figure 5. Differential energy spectrum obtained from the stacked source analysis. It is well described by a power law with index $-3.16 \pm 0.51$. The integral flux above $150 \mathrm{GeV}$ corresponds to $1.5 \%$ of the flux of the Crab Nebula. The spectrum of the Crab Nebula is shown as a dashed gray line.

with the energy bin width $\Delta E_{i}$. The mean energy spectrum in the observer's frame for all 21 blazars considered in the stacking analysis can be well described by a power law

$$
\frac{d N}{d E}=(2.5 \pm 0.7) \times 10^{-11} \frac{1}{\mathrm{TeV} \mathrm{cm}^{2} \mathrm{~s}} \cdot\left(\frac{E}{E_{0}}\right)^{-3.16 \pm 0.51}
$$

with $E_{0}=200 \mathrm{GeV}$. The differential flux at $200 \mathrm{GeV}$ corresponds to $1.9 \%$ of the one for the Crab Nebula. The integral flux above $150 \mathrm{GeV}$ is determined as $F=4.3 \times 10^{-12} \mathrm{~cm}^{-2} \mathrm{~s}^{-1}$ corresponding to $1.5 \%$ of the integral Crab Nebula flux above $150 \mathrm{GeV}$.

On average, each blazar contributes $(2.1 \pm 0.3) / \mathrm{hr}$ excess events to the cumulative excess as illustrated in Figure 4. The objects are ordered in right ascension.

In Figure 5, the measured spectrum is shown.

\section{DISCUSSION}

The positive mean significance distribution indicates that the X-ray selected blazars studied here constitute a fairly representative sample of generic VHE emitters, as suggested by Costamante \& Ghisellini (2002). The recent discoveries of individual blazars from the sample indeed corroborate this finding. The next generation of Cherenkov experiments-MAGIC-II, HESS 2, and later on the Cherenkov Telescope Array (CTA; Wagner et al. 2009) - will therefore have good chances to detect an increasing fraction of all known X-ray blazars.

\subsection{Gamma-ray Background}

At $200 \mathrm{GeV}$, the attenuation caused by the EBL is negligible, according to the model of Kneiske \& Dole (2010), so the calculation of the broadband spectral index $\alpha_{X-\gamma}$ between $1 \mathrm{keV}$ and $200 \mathrm{GeV}$ can be done with the observed VHE energy spectrum. The mean energy flux at $200 \mathrm{GeV}$ is calculated from the fit to $1.60 \times 10^{-12} \mathrm{erg} \mathrm{cm}^{-2} \mathrm{~s}^{-1}$. This value is compared to the mean X-ray energy flux at $1 \mathrm{keV}$ for all sources, weighted with their individual observation time, which is $3.74 \mu \mathrm{Jy}$, corresponding to a flux of $9.05 \times 10^{-12} \mathrm{erg} \mathrm{cm}^{-2} \mathrm{~s}^{-1}$. The ratio 


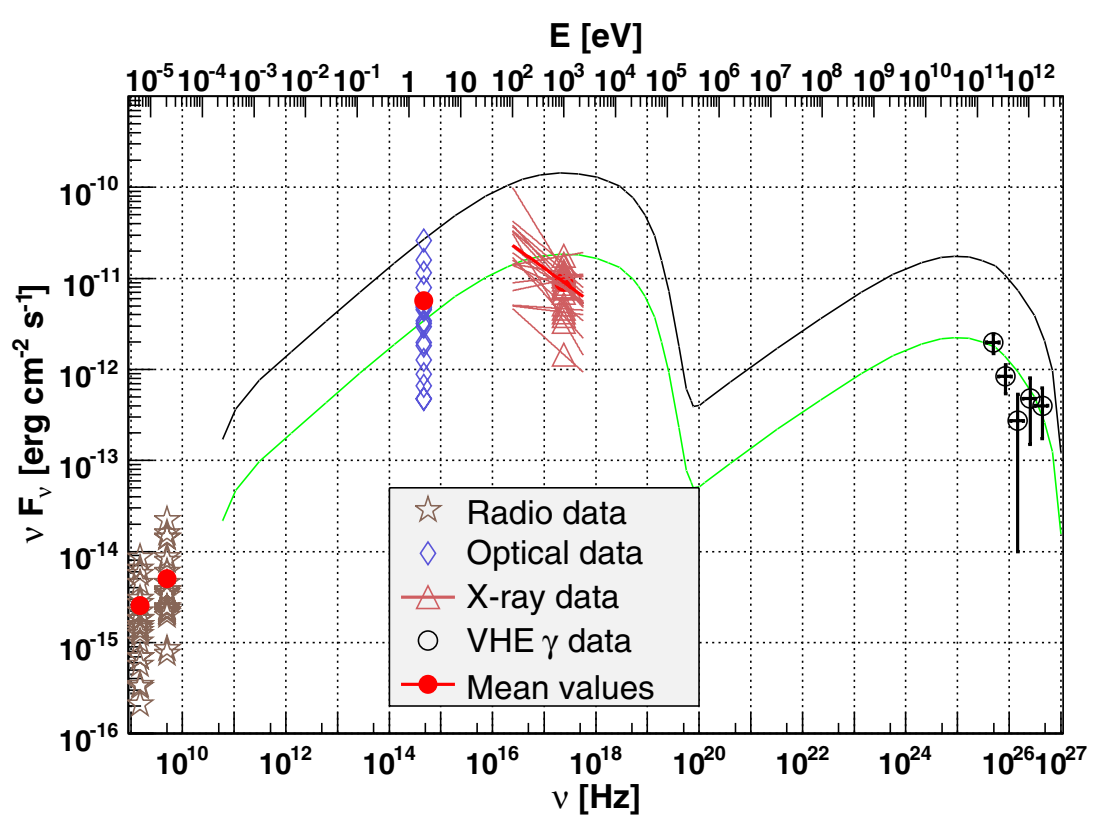

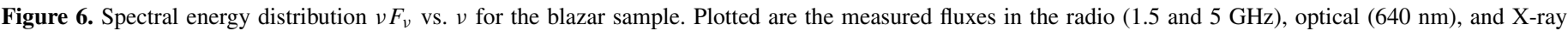

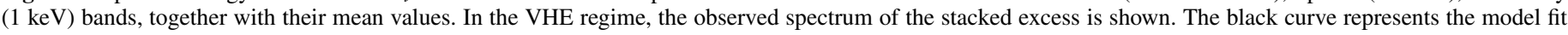

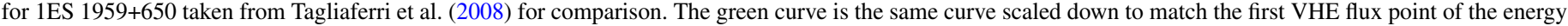
spectrum of the stacked excess.

of X-ray (1 keV) to $\gamma$-ray $(200 \mathrm{GeV})$ flux is

$$
\frac{v F_{v}(1 \mathrm{keV})}{v F_{v}(200 \mathrm{GeV})}=5.66
$$

resulting in a broadband spectral index $\alpha_{X-\gamma}=1.09$.

The result suggests that during quiescence the $\mathrm{X}$-ray luminosity is higher than the VHE $\gamma$-ray luminosity above $200 \mathrm{GeV}$. Here, we tacitly assume that the X-ray data, which are not contemporaneous with the $\gamma$-ray data, are representative of the baseline emission as well. Note that the X-ray as well as the VHE data are averages over the whole blazar sample considered here and that flux variations commonly observed with the $\mathrm{X}$-ray band do not influence $\alpha_{X-\gamma}$ across eight orders of magnitude. A simple estimation of $\Delta \alpha_{X-\gamma}$ by inferring the error of the average value at $1 \mathrm{keV}$ of the sample and the error of the energy spectrum at $200 \mathrm{GeV}$ results in $\Delta \alpha_{X-\gamma}=0.04$. With the newly found X-ray to $\gamma$-ray spectral index of $\alpha_{X-\gamma}=1.09$ one can infer the luminosity function of VHE blazars from their X-ray luminosity function, avoiding the bias toward flares. Assuming equal X-ray and VHE $\gamma$-ray luminosities, HBLs already fail to explain the extragalactic diffuse $\gamma$-ray background (Kneiske $\&$ Mannheim 2008).

\subsection{Spectral Energy Distribution}

As no flaring activity has been seen on diurnal scales or on longer timescales, the cumulative signal of the high-peaked blazars in this sample can be accounted for as an upper limit on their baseline emission in VHE $\gamma$-rays, although variability on flux scales below the sensitivity limit of MAGIC may not be excluded.

The SED for the blazar sample is determined by taking archival data in the radio and $\mathrm{X}$-ray bands $(1.4 \mathrm{GHz}, 5 \mathrm{GHz}$, and $1 \mathrm{keV})$ if available as well as contemporaneous optical data in the $R$ band $(640 \mathrm{~nm})$ taken with the KVA telescope. The collected data are shown in Figure 6. In the VHE regime also, the deabsorbed spectrum as already shown in Figure 5 is displayed. From the mean values in optical and X-rays and the mean X-ray spectral index, one can infer an average synchrotron peak energy of the sample below $1 \mathrm{keV}$.

For a simple comparison, the measured SED of the HBL 1ES $1959+650$ is drawn. 1ES $1959+650$ is a well-known VHE blazar which was observed in a historic low emission state in a multiwavelength campaign in 2006 (Tagliaferri et al. 2008). The differential energy spectrum measured by MAGIC in the VHE regime follows a power law with a photon index $\Gamma=-2.58 \pm 0.18$. The SED of 1ES $1959+650$ can be well fitted with a one-zone synchrotron self-Compton model, which is also plotted in Figure 6. To guide the eye, the SED is also scaled down to the lowest energy bin of the VHE spectrum of the blazar sample.

\section{CONCLUSIONS}

In the course of the MAGIC observational program during 2004-2009, a major part was spent on X-ray bright BL Lacertae objects. For 21 non-detections, upper limits on the integral flux ranging between $1.6 \%$ and $13.6 \%$ of the Crab Nebula flux could be determined. Applying a stacking method to the individual non-detections, we found an average VHE emission of the sample of X-ray selected blazars at the $4.9 \sigma$ significance level above $100 \mathrm{GeV}$. It turns out out that the mean VHE $\gamma$-ray flux is significantly lower than in archival X-ray measurements. The two-point spectral index between $1 \mathrm{keV}$ and $200 \mathrm{GeV}$ is $1.09 \pm 0.04$.

We would like to thank the Instituto de Astrofisica de Canarias for the excellent working conditions at the Observatorio del Roque de los Muchachos in La Palma. The support of the German BMBF and MPG, the Italian INFN, the Swiss National Fund SNF, and the Spanish MICINN is gratefully acknowledged. This work was also supported by the Polish MNiSzW Grant N N203 390834, by the YIP of the Helmholtz 
Gemeinschaft, and by grant DO02-353 of the Bulgarian National Science Fund.

\section{REFERENCES}

Acciari, V., et al. 2009, ApJ, 690, L126

Acciari, V., et al. 2010, ApJ, 708, L100

Aharonian, F., et al. 2000, ApJ, 539, 317

Aharonian, F., et al. 2006, A\&A, 457, 899

Aharonian, F., et al. 2007a, ApJ, 664, L71

Aharonian, F., et al. 2007b, A\&A, 475, L9

Albert, J. 2007, ApJ, 669, 862

Albert, J., et al. 2008a, ApJ, 681, 944

Albert, J., et al. 2008b, Nucl. Instrum. Methods Phys. Res. A, 594, 407

Albert, J., et al. 2008c, ApJ, 674, 1037

Aliu, E., et al. 2009, Astropart. Phys., 30, 293

Benbow, W. 2009, arXiv:0908.1412

Bretz, T. 2005, in AIP Conf. Proc. 745, 2nd Intl. Symp. High Energy Gammaray Astronomy, ed. F. A. Aharonian, H. J. Völk, \& D. Horns (Melville, NY: AIP), 730

Bretz, T., \& Dorner, D. 2008, in AIP Conf. Proc. 1085, Proc. 4th Intl. Mtg. High Energy Gamma-ray Astronomy, ed. F. A. Aharonian, W. Hofmann, \& F. Rieger (Melville, NY: AIP), 664

Bretz, T., \& Wagner, R. 2003, in Proc. 28th ICRC (Tsukuba), Vol. 5, 2947

Costamante, L., \& Ghisellini, G. 2002, A\&A, 384, 56
Dermer, C. D., \& Schlickeiser, R. 1993, ApJ, 416, 458

Donato, D., Ghisellini, G., Tagliaferri, G., \& Fossati, G. 2001, A\&A, 375, 739

Giommi, P., Piranomonte, S., Perri, M., \& Padovani, P. 2005, A\&A, 434, 385

Grube, J. 2007, in Proc. 30th ICRC (Merida), Vol. 2, 691

Hillas, A. M. 1985, in Proc. 19th ICRC (La Jolla), Vol. 3, 445

Hofmann, W., \& Fegan, S. 2009, ATel, 2293, 1

Kneiske, T. M., \& Dole, H. 2010, A\&A, 515, 19

Kneiske, T. M., \& Mannheim, K. 2008, A\&A, 479, 41

Lessard, R. W., Buckley, J. H., Connaughton, V., \& Le Bohec, S. 2001, Astropart. Phys., 15, 1

Li, T., \& Ma, Y. 1983, ApJ, 272, 317

Mannheim, K. 1993, A\&A, 269, 67

Mannheim, K., Westerhoff, S., Meyer, H., \& Fink, H.-H. 1996, A\&A, 315, 77

Maraschi, L., Ghisellini, G., \& Celotti, A. 1992, ApJ, 397, L5

Muecke, A., \& Protheroe, R. J. 2001, Astropart. Phys., 15, 121

Nieppola, E., Tornikoski, M., \& Valtaoja, E. 2006, A\&A, 445, 441

Ong, R. A., \& Fortin, P. 2009, ATel, 2272, 1

Riegel, B., Bretz, T., Dorner, D., Berger, K., \& Höhne, D. 2005, in Proc. 29th ICRC (Pune), Vol. 5, 215

Rolke, W., Lopez, A., \& Conrad, J. 2005, Nucl. Instrum. Methods Phys. Res. A, 551, 493

Sikora, M., Begelmann, M. C., \& Rees, M. J. 1994, ApJ, 421, 153

Stecker, F. W., de Jager, O. C., \& Salamon, M. H. 1996, ApJ, 473, L75

Tagliaferri, G., et al. 2008, ApJ, 679, 1029

Teshima, M. 2009, ATel, 2098, 1

Wagner, R. M., Lindfors, E. J., Sillanpää, A., \& Wagner, S. 2009, arXiv0912.3742 